J Electron Microsc Tech. 1986 ; 3(2): 233-241. doi:10.1002/jemt.1060030210.

\title{
A Simple Method for Quick-Freezing
}

\author{
ELAINE L. BEARER and LELIO ORCI \\ Department of Pathology, University of California, San Francisco, CA 94143 (E.L.B.); Institute \\ d'Histologie, Department de Morphologie, Centre Medicale Universitaire, Geneva 1211, \\ Switzerland (L.O.)
}

\section{Abstract}

In conventional freeze-fracture replicas produced from tissue cryoprotected with glycerol, the hydrophobic inner surfaces of membranes are revealed, but hydrophillic structures are obscured in the surrounding ice. Quick-freezing of tissue obviates the need for glycerol, which prevents the removal of this ice by etching or freeze-drying, but the major problem in freezing without glycerol cryoprotection is ice crystal formation. We describe here a simple method for quick-freezing tissue, in the absence of glycerol, on a nitrogen-cooled copper block with a hand-held specimen holder. This method freezes samples well enough to preserve molecular detail that can be revealed by subsequent etching. We show some examples of the quality of this freezing with respect to the visualization of molecular detail in isolated protein molecules such as ferritin and catalase.

Furthermore, we show examples of in situ cellular structures that are revealed by this method, and we compare the structure seen in these replicas with structures preserved by quick-freezing at liquid helium temperatures.

\section{Keywords}

Quick-freezing; Deep-etching; Balzers' freeze fracture device

\section{INTRODUCTION}

This report describes an inexpensive and simple method for the rapid freezing of either fresh or chemically fixed tissue, which can then be either fractured for freeze-fracture replication with or without etching, or freeze-substituted and embedded for thin-sectioning. We developed this technique because liquid helium, used in the Heuser et al. method (1972), was both prohibitively expensive and difficult to obtain. With this modified method, we were able to prepare specimens comparable to those frozen on a helium-cooled copper block by using liquid nitrogen. The success rate (the number of well frozen specimens compared to the number of attempts) was much higher with helium, but well frozen samples from either method were comparable with respect to the preservation of structural detail.

Glycerol, the standard cryoprotectant used in the preparation of freeze-fracture, has been shown to change lipid membrane structure (Bearer et al., 1982) and prohibits water 
sublimation from a fractured, frozen surface. Hence, freezing tissue in its absence has great advantages. However, it is extremely difficult to avoid extensive ice crystal formation and consequent structural damage when freezing specimens without glycerol cryoprotection (see review by Gilkey, 1985). Freezing of noncryoprotected material by immersion in cold liquid gasses is difficult because the contact with warm tissue heats the cold gas, which goes into the gaseous phase. The expansion that occurs during the liquid-to-gas-phase transition destroys tissue ultrastructure. The gas becomes too warm to freeze adequately immediately adjacent water, which crystallizes and also damages the tissue.

The most successful methods of quick-freezing use a super-cooled metal block to avoid direct contact of the tissue with the liquid gas, or a jet spray of liquid gas, which rapidly and continuously replaces warmed gas. For metal-block freezing, copper is the preferred metal since it is the best conductor, and hence can absorb and dissipate the heat energy of the tissue quickly.

The rate of cooling must be rapid, as the goal is to capture both fine structure and water in its fluid state. Hence, sudden impact on a super-cooled copper block, highly polished both to insure equal contact of the tissue across the block surface and to maximize conductivity of heat energy, has proven to be the most effective method (Heuser et al., 1972). Liquid helium was chosen to cool the block since its temperature is the coldest of all liquid gasses, $2-4^{\circ} \mathrm{K}$. Thus, the most rapid rate of freezing is obtained owing to the largest differential between ambient and copper block temperatures. Liquid helium, however, is dangerous to use. This is because its extremely low temperature has the potential for freezing oxygen from the atmosphere and hence concentrating it. Thus, helium requires a complicated system of valves and tubing to use. Furthermore, it has recently been shown that the specific heat of copper is reduced by a factor of $2 \times 10^{3}$ when it is cooled from liquid nitrogen to liquid helium temperature. This means that the copper may be more efficient at dissipating the heat from the tissue at liquid nitrogen rather than liquid helium temperatures (Bald, 1983). We used liquid nitrogen to cool the block, and a simple, hand-held epoxy-headed hammer to bring the tissue into contact with the block. Instead of glycerol, we used methanol to aid in the prevention of ice crystal formation as well as to accelerate subsequent etching of the fractured surface. By this method we have examined purified proteins-catalase and cationic ferritin —as well as gluteralde-hyde-fixed whole tissue blocks, cell suspensions, and monolayers.

\section{MATERIALS AND METHODS}

We used an electronic-grade copper cylinder, $99.99 \%$ pure, $4 \mathrm{~cm}$ in diameter, and $6.2 \mathrm{~cm}$ in height (Fig. 1A \& B). The center of the cylinder was reamed out to within $0.5 \mathrm{~cm}$ of the sides and $2.2 \mathrm{~cm}$ of the top. Four additional holes, $1.0 \mathrm{~cm}$ in diameter, were drilled at $90^{\circ}$ intervals around the sides of the block and centered $2.2 \mathrm{~cm}$ below the top. After all holes had been drilled, the copper cylinder weighted $410 \mathrm{~g}$. The surface was polished mirror-smooth and cleaned with wenol ${ }^{\circledR}$, sonicated in acetone, and rinsed in $90 \%$ ethanol. The cylinder was placed standing up in a 12-in square, 6-in deep styrofoam box, and a lead weight inside an aluminum cup measuring slightly larger than the flat surface of the cylinder was placed on top of the cylinder to minimize recondensation. The styrofoam box was filled with liquid 
nitrogen to within $2 \mathrm{~cm}$ of the top of the cylinder. The nitrogen was continuously added to the box, but it was never allowed to overflow the cylinder surface, until it stopped boiling. At that point the specimens were prepared, and the block was allowed to equilibrate to the liquid nitrogen temperature. This usually took about 10 minutes. The complete set-up is shown in Figure 1A.

We used rat tissue that was fixed by intra-cardiac perfusion of $1.5 \%$ gluteralderhyde in 0.1 $\mathrm{M}$ sodium cacodylate, $\mathrm{pH} 7.4$ solution and then chopped into $0.5-\mathrm{mm}$ square blocks. These were incubated for 20 minutes to 1 hour prior to freezing in $20 \%$ methanol in phosphate buffered saline (PBS). Other buffers made no difference in freezing quality. We also used cationic ferritin (Miles Laboratory, PA) and ox liver catalase (Sigma, MN) suspended just before freezing (5-10 $\mathrm{min})$ in. 20\% methanol in PBS. These later solutions were used directly from the bottle and diluted 1:10 in the methanol solution.

This suspension was either frozen directly, or mixed with mica chips (Heuser, 1983) and then frozen as a droplet. Aliquots $(20 \mu \mathrm{l})$ of suspensions of the purified proteins were placed on the same supports by means of Eppendorff micropipettes. The gold supports were stuck onto double-stick, foam padded $\operatorname{scotch}^{\circledR}$ tape, which had been put onto the epoxy-head of a small hammer. Just before freezing, any extra liquid on the surface of tissue block specimens was removed by touching them lightly with Whatman No.1 filter paper, so that only a small miniscus of fluid around the periphery between tissue and support was allowed to remain. In a single motion, the lead-aluminum cover was lifted off the block with the gloved left hand, and the right hand brought the hammer down gently onto the copperblock, allowing it to rest there for ten seconds. Then the double-stick tape-also frozen-was pulled off the hammer head as the hammer was lifted, and tape and specimens were immediately submerged in liquid nitrogen. The specimens were examined while immersed in the nitrogen to see if they retained their unfrozen color and were transparent. Any specimens appearing opacified or whitened were discarded.

The block was cleaned after three to four freezing times by three-second sonication in acetone, a 70\% ethanol rinse, and blow-drying. It was reusable three to four times, provided different areas were used to actually contact the tissue.

\section{Fracture and etch}

We froze specimens immediately before mounting them into the Balzers Bell jar onto the rotary stage, which had been allowed to settle at $-150^{\circ} \mathrm{C}$ for at least 15 minutes prior to mounting the specimens.

We noted that frozen acetone (melting point $-95^{\circ} \mathrm{C}$ ) remained frozen up to $65^{\circ} \mathrm{C}$ on the gauge. We also noted that frozen methanol sublimed during the pumpdown of the bell jar and before we could observe its melting point on the Balzers temperature gauge. Hence, we maintained a temperature of $-150^{\circ} \mathrm{C}$ while achieving a vacuum of $10^{-7}$ torr, and only began to warm the table just before fracturing. We fractured all samples at $-130^{\circ} \mathrm{C}$ on the gauge.

Fracturing of only the uppermost layer of tissue was achieved by carefully scraping the ice off the sample and then lowering the knife $0.5 \mu \mathrm{m}$ by the mechanical attachment, or one 
quarter turn by hand crank, and scraping the tissue once. When the fractured tissue surface was observed, as for unetched specimens, it was mirror-smooth and opalescent. For etching, the knife was left over the specimen after this single fracture while warming the specimen table to $-75^{\circ} \mathrm{C}$ by the Balzer's gauge (clearly colder than $-95^{\circ} \mathrm{C}$ in real temperature, as the acetone did not melt). Twenty minutes was allowed to elapse between fracture and shadowing. The specimens were periodically checked by briefly moving the knife. The meniscus of liquid around the periphery could be seen to recede. We found that if the meniscus totally disappeared, apparent cold contact between stage and tissue was lost and ice recrystalization occurred. On at least one out of every five runs, the fluctuations in temperature of the Balzers stage also elicited ice recrystalization, destroying the sample. The lack of stability of the temperature of the Balzer's stage has been previously reported (Ruben, 1985).

\section{The shadow}

We used electron guns, the carbon electrode immediately above the stage, and the platinum set at a $22^{\circ}$ angle. The stage rotated at a speed of approximately $60 \mathrm{rpm}$. We did not use a quartz crystal monitor but instead fired the guns at the lowest voltage $(1,500 \mathrm{~V})$ and emission (55-60 mA) to give evaporation for 18 seconds, so that the shadow was not thicker than 1-2 nm as measured on the replica by Heuser's method (1983). This method, in brief, consists of the following: the width of the replica of a particle is measured, then the width of two similar particles close enough together to obscure the definition between them is measured, then the difference between them, divided by two, is obtained. This difference equals the thickness of the shadow. We found that a single platinum plug could be fired five to seven times, with similar results, if fired at the same voltage settings and for the same length of time. The first and last firing were often thicker or thinner. The carbon gun was fired without rotation at a voltage of $1,100 \mathrm{~V}$ and emission current of 180-190 A.

The replicas were cleaned by floating in Eau de Javel (2.5\% hypochlorite) for $45 \mathrm{~min}$, swirling in three changes of water, swirling for five to ten minutes in methanol: chloroform (1:2 ratio), and finally washing in water. The methanol-chloroform mixture was added slowly to the porcelain dish, as it violently attacks the fragile replica. Replicas of icedamaged tissue were frequently recognized during cleaning, as they were much more fragile than well-frozen samples.

\section{Freeze substitution}

Freeze substitution was performed by freezing on the copper block as described above, followed by immediate submersion in a 20-cc scintillation counter glass jar containing $2 \mathrm{cc}$ of 5\% osmium tetroxide in acetone and filled with liquid nitrogen. The jars were then moved to a styrofoam box filled with dry ice and $100 \%$ alcohol, and allowed to thaw to $-78^{\circ} \mathrm{C}$ (the temperature of dry ice). After two days, they were sequentially warmed to $-20^{\circ} \mathrm{C}$ in the freezer for one hour, then $4^{\circ} \mathrm{C}$ for one hour. Then, at room temperature, the acetone-osmium was replaced by $10 \%$ uranyl acetate in $100 \%$ ethanol and incubated for 1 hour at room temperature. Then the specimens were further dehydrated twice in propylene oxide and embedded in Epon 812. Silver grey thin sections were picked up on parlodion-copper grids and poststained with aqueous uranyl acetate and lead citrate. 
Negative staining was performed by wetting a parlodion-coated, carbon-shadowed copper grid with a suspension of catalase, gently withdrawing the fluid by touching the grid with a damp filter paper until only a surface sheen remained, and then immediately placing the grid face down on a drop of $1 \%$ phosphotungstic acid for 1 minute. Again, the side of the grid was gently touched with damp filter paper until only a grey film remained. The grids were then allowed to air dry for at least one hour before they were put into the microscope. Electron micrographs were made on Phillips 300 and JEOL CX100 microscopes.

\section{RESULTS}

Cationic ferritin, frozen at nitrogen temperature, displayed the same structure as has been described previously by Heuser (1983) for helium-cooled specimens (Fig. 2A). Catalase crystals, frozen as a suspension, displayed remarkable detail and were more informative at higher magnifications than their negatively stained counterpart (Fig. 2B, C).

Tissue blocks revealed extracellular or cell surface structures that had been submerged by the ice in glycerinated fractures. The endothelial fenestral diaphragm, a water-filled channel, was bared by etching (Fig. 3A). Because the material was fractured, all surfaces of the cell membrane as well as intramembranous fracture faces could be viewed. The diaphragms consisted of radial fibrils, which arose from the pore's brim symmetrically and interlaced as they traversed the pore's center to reinsert in the brim. The intervening wedge-shaped channels averaged $5.65 \mathrm{~nm}$ in maximal arc width, as we have reported (Bearer and Orci, 1985). Glomerular capillary fenestrae could be seen to be devoid of diaphragmatic structures (Fig. 3B). Instead, the dense fibrillar texture of the glomerular basement membrance was revealed through the pores. Decorations of these pores in freeze-etch replicas by cationic ferritin confirmed their position within the endothelial pores. Thus, they were proved to be the equivalent of the fenestral diaphragm already well described in thin-sections (reviewed in Simionescu, 1983; and Simionescu et al., 1981) (Fig. 3C, D).

Intracellular structure was less well revealed by deep-etching of whole, unextracted tissue. The basal region of the kidney tubular epithelial cells displayed a marked amount of intramembranous particles in the protoplasmic fracture face (P-face) after etching. External surface views of this same membrane displayed a dense hexagonal array of particles, reminiscent of the pattern seen on the bladder surface. Peroxisomes displayed a striking crystalline pattern on the E-fracture face, which had been seen only faintly in traditional fractures (Kalmbach and Fahimi, 1978) (Fig. 4B).

Freeze-substitution of kidney cortex, frozen as if for freeze-fracture on the copper block but subsequently embedded in acetonic osmium, revealed a uniformly dense cytoplasm. Nuclei, the most succeptible intracellular organelle to ice crystal formation, were without the diagnostic lucencies caused by water crystallization to within several microns of the surface that had contacted the copper block. A well-preserved nucleus (Fig. 5A) is compared with a nucleus from deeper in the tissue displaying characteristic ice-crystal deformation (Fig. 5B). Quick-freezing on a helium-cooled copper block in the Hitech apparatus displayed identical structures within the endothelial fenestral pore as those seen in preparations that used a nitrogen-cooled copper block (Fig. 5C). 


\section{DISCUSSION}

Quick-freezing on a nitrogen-cooled copper block is a practical possibility. The replicas obtained rival in quality those produced by helium-cooled-block freezing. The major drawback is that a lower success rate is achieved with the hand-held nitrogen-cooled device than with an automated helium device. This lower rate may be more due to the variability of manual mechanics versus the precision of automated mechanics rather than to the cryogenic properties of nitrogen versus helium, since well-frozen samples are reproducible with other automated devices that use nitrogen (Phillips and Boyne, 1984; Dudek et al., 1984). However, the method is so simple to set up and perform that simple steps to check freezing quality before investing in the time required for Balzer's fracture-etch replication can eliminate some of the time wasted on inadequately frozen samples. These steps include monitoring the color and transparency of the frozen tissue, along with the use of methanol for cryoprotection.

Freeze-etching has produced elegant visions of hitherto invisible structures (Heuser et al., 1972). Etching has also produced greater resolution of some molecules than either negativestaining or heavy-metal-staining of thin sections, since neither charge-induced collapse, superimposition of two sides of a three dimensional structure, nor affinity for the stains is operative. Quick-freezing itself is useful to capture cellular events within milliseconds of their occurence (Chandler and Gilkey, 1985). Such capture is impossible with chemical fixation, which is by no means instantaneous.

Quick-freezing as demonstrated here allowed the sublimation of water after fracturing, which then revealed a physiologically significant structure, the fenestral diaphragm. The geometric shape of the channel thus revealed beneath the ice can be used to generate equations predicting permeability properties of fenestrated endothelia previously impossible to define. Ice formation, a major source of artifact, was shown to be absent in freezesubstituted samples. Furthermore, labeling with cationic ferritin tells us that the diaphragmetic structures (1) are not produced by ice formation artifact and (2) indeed occupy the fenestral diaphragm as previously defined by thin-section. Internal membranes and cytoplasmic skeletal structures were less well visualized in fractured and etched whole tissue than in monolayers lysed open and washed in distilled water. Isotonic salt concentrations preserved structure better than distilled water and did not leave a residue that obscured fine detail. Molecular structure of globular or crystalline proteins could be visualized with the same resolution as with helium freezing. As has already been shown (Heuser, 1983), molecular detail was better preserved by deep-etching than by negativestaining.

We believe that many more structures await elucidation by the simple method presented here.

\section{Acknowledgments}

The authors thank Patrick Sors for his superb technical assistance that made this project possible, Dr. Dan Friend for many inspiring discussions, and Merle Jolson for invaluable secretarial help.

This project was supported by Swiss National Science Foundation grant 3.460.83.

J Electron Microsc Tech. Author manuscript; available in PMC 2015 November 06. 


\section{References}

Bald WB. Optimizing the cooling block for the quick-freeze method. J Microsc. 1983; 131:11-23. [PubMed: 6887237]

Bearer EL, Duzgunes N, Friend DS, Papahadjopoulos D. Fusion of phospholipid vesicles arrested by quick-freezing. The question of lipidic particles as intermediates in membrane fusion. Biochim Biophys Acta. 1982; 693:93-98. [PubMed: 7150597]

Bearer EL, Orci L. Endothelial fenestral diaphragms: A quick-freeze, deep-etch study. J Cell Biol. 1985; 100:418-428. [PubMed: 3968170]

Chandler D, Heuser JE. Arrest of membrane fusion events in mast cells by quick-freezing. J Cell Biol. 1980; 86:666-674. [PubMed: 7400221]

Dudek RW, Boune AF, Charles TM. Novel secretory granule morphology in physically fixed pancreatic islets. J Histochem Cytochem. 1984; 32:924-934.

Gilkey J. Freezing tissue review. J Electron Microsc Tech. 1985 (in press).

Heuser JE. Procedure for freeze-drying molecules adsorbed to micaflakes. J Mol Biol. 1983; 169:155195. [PubMed: 6684695]

Heuser JE, Reese TS, Landis DM. Preservation of synaptic structures by rapid freezing. Cold Spring Harbor Symp Quant Biol. 1972; 40:17-24. [PubMed: 1065523]

Kalmbach P, Fahimi MD. Peroxisomes: Identification in freeze-etch preparations of rat kidney. Cell Biol Intl Rep. 1978; 2:389-396.

Phillips TW, Boyne AF. Liquid nitrogen-based quick freezing. J Electron Microsc Tech. 1984; 1:9-29.

Ruben GC. A comparison of specimen stage surface temperature and the G.A.-1 control unit reading in Balzer's freeze fracture microtome cold stage. J Electron Microsc Tech. 1985; 2:53-57.

Simonescu N. Cellular aspects of transcapillary exchange. Physiol Rev. 1983; 6:1536-1577.

Simionescu M, Simionescu N, Silbert JE, Palade GE. Differentiated microdomains on the luminal surface of the capillary endothelium. II Partial characterization of their anionic sites. J Cell Biol. 1981; 90:614-621. [PubMed: 6457053] 

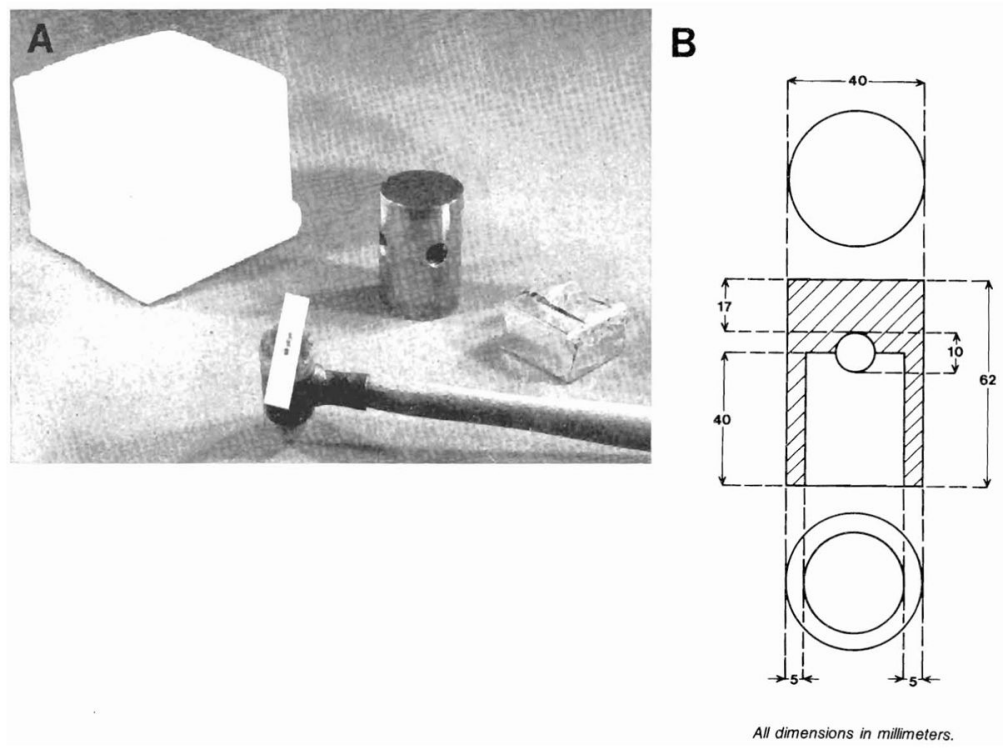

Fig. 1.

Quick-freeze apparatus: A. White styrofoam box, electronic grade copper cylinder with highly polished surface, aluminum-covered weight to cover the cylinder during cooling down, and an epoxy-headed hammer with three gold specimen supports mounted on the hammer with double-stick insulation tape. B. The dimensions of the copper cylinder and the placement of the holes drilled into it. 

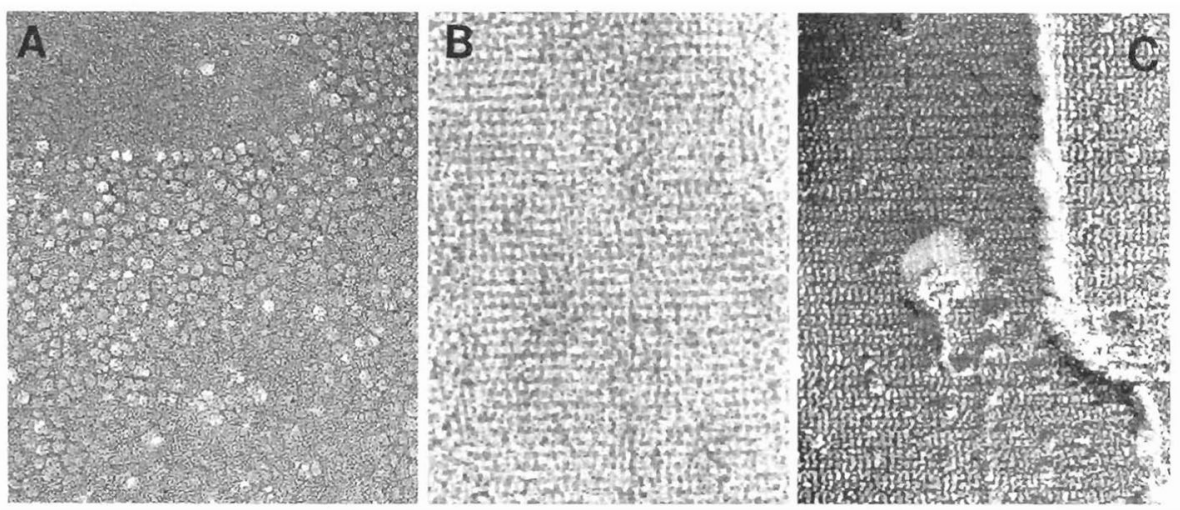

Fig. 2.

A. Freeze-etched cationic ferritin mixed with mica chips and frozen on the cylinder cooled to liquid nitrogen temperatures $(\times 140,000)$. B. Catalase crystals negative-stained with $1 \%$ phosphotungstic acid $(\times 200,000)$. C. Catalase crystals quick-frozen on the nitrogen-cooled copper cylinder and etched before rotary shadowing $(\times 200,000)$. 


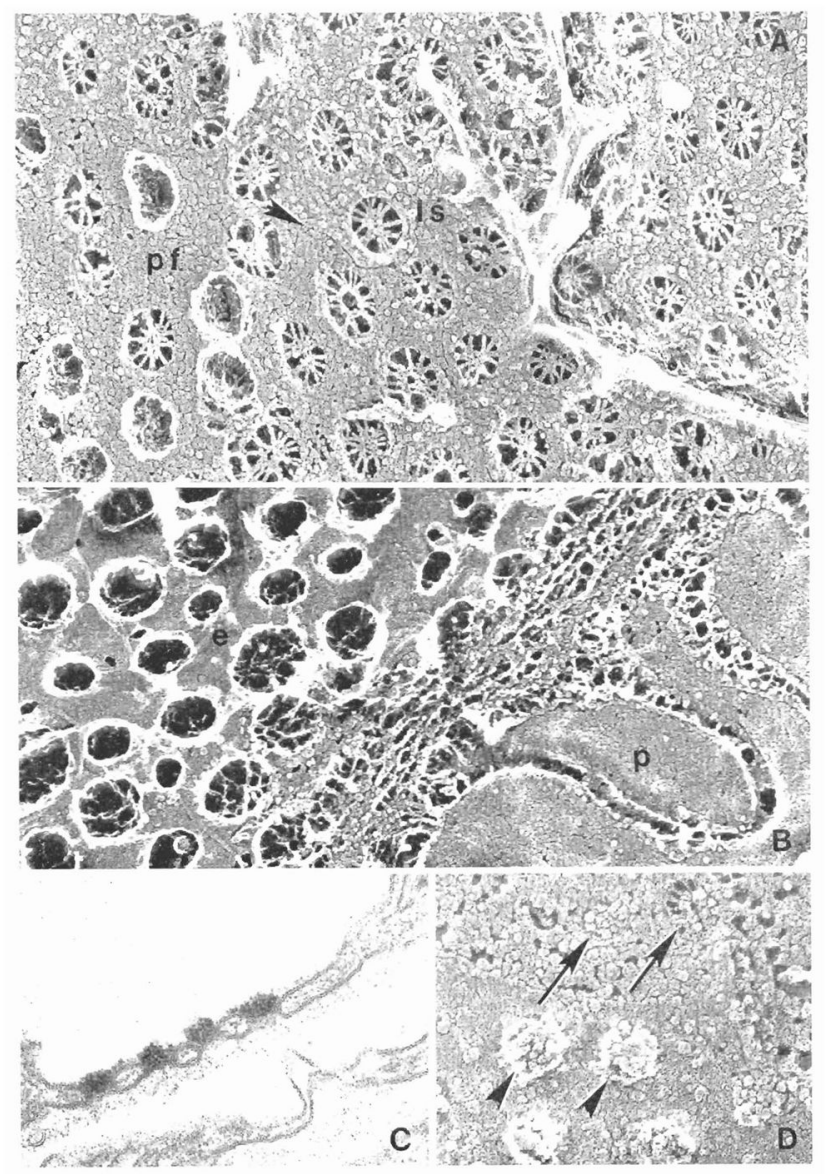

Fig. 3.

A. A view of the flower-like endothelial fenestral diaphragms on the lumenal surface (1s) of the rat kidney peritubular capillary and in the protoplasmic fracture face $(\mathrm{pf})$ of the lumenal membrane. The fracture line (arrowhead) can be seen separating surface and intramembraneous views. Some P-face pores lack diaphragms. These are probably torn out with the E-face during the fracturing procedure $(\times 160,000)$. B. The fenestral pores in the glomerular capillary of the rat kidney have no diaphragms. Instead the basement membrane that separates endothelial cell from podocyte foot processes $(\mathrm{p})$ can be discerned within. The blank pores $(\mathrm{e})(\times 120,000)$. C. Thin-sections of animals perfused with cationic ferritin; capillary fenestral diaphragms are heavily labeled by the electron-dense core of the ferritin whereas adjacent membrane is not $(\times 100,000)$. D. After freeze-etching, heaps of cationic ferritin are present on the lumenal surface of peritubular capillaries. These mounds of particles (arrowheads) have the same regular distribution as the fenestral diaphragms that they obscure. In the P-fracture face, the mounds are partially broken away, and the underlying diaphragmatic spokes can be seen beneath the cationic-feritin particles (arrows) $(\times 130,000)$. 


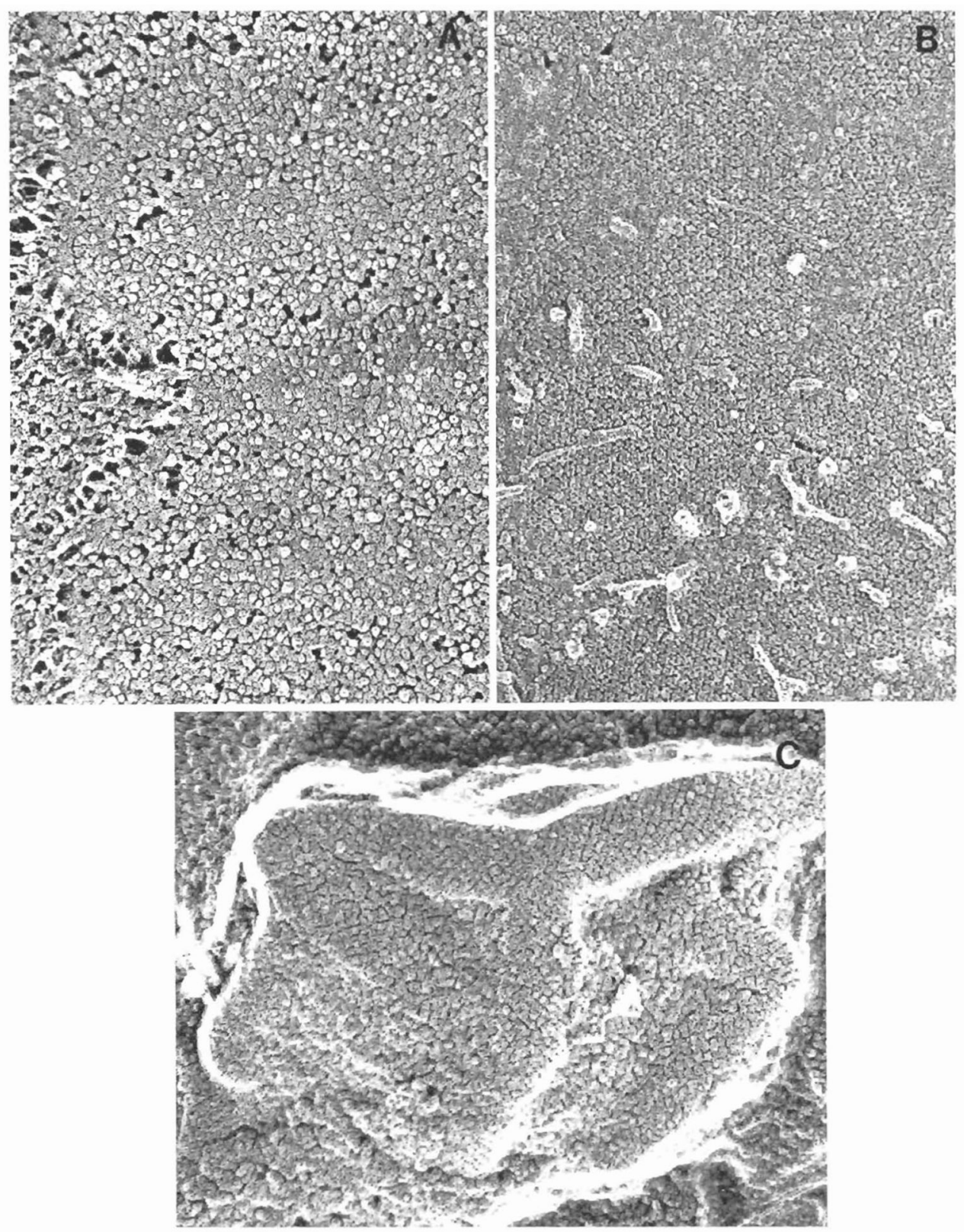

Fig. 4.

A. The P-fracture face of the basal membrane in kidney tubular epithelia is very densely populated by particles after etching. The overlying basement membrane can bee seen along the left border of the micrograph $(\times 140,000)$. B. The true surface of the same basal region of the plasma membrane, showing large filamentous structures that may have attached the cell to the basement membrane, and a fine hexagonal array of surface particles $(\times 140,000)$. C. Intracellular organelle; the microbody displays the crystalline pattern of its E-face membrane after etching $(\times 220,000)$. 


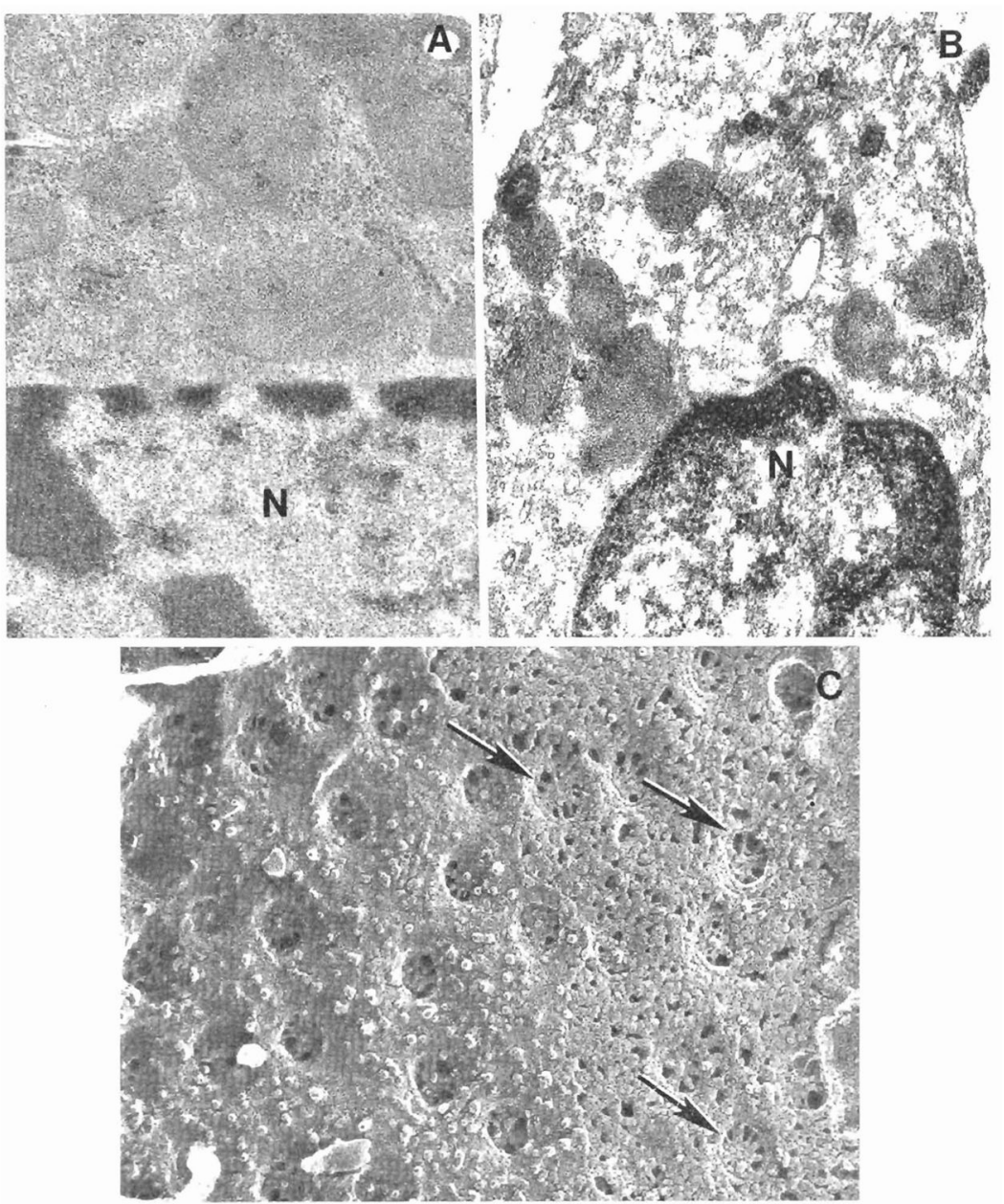

Fig. 5.

A, B. Freeze substitution of quick-frozen noncryoprotected samples is a good way to check for the presence of distorting ice crystal formation. In A, after nitrogen-cooled-copper-block freezing, the cell is well-frozen, as evidenced by the uniformly grey cytoplasm. The nucleus (N) is also unaffected by ice. However, deeper in the same tissue block (B) the cytoplasm shows large, irregular clearings, which suggest the presence of ice crystalization during freezing (A, $\times 45,000 ; \mathrm{B}, \times 45,000)$. C. Quick-freezing on the helium-cooled copper block without methanol shows the same fenestral diaphragmatic structures (arrows) as those specimens frozen by hand on the nitrogen-cooled block. The P-face membrane (the right half of the micrograph) has more holes (black defects) than the hand frozen tissue $(\times 130,000)$. 